\title{
Cystic adventitial disease: A cause of peripheral arterial disease that should be remembered in young adults
}

\author{
Erdinç Eroğlu (D), Alptekin Yasım (iD, Mehmet Acıpayam (D) \\ Department of Cardiovascular Surgery, Kahramanmaraş Sütçü İmam University Faculty of Medicine, Kahramanmaraş, Turkey
}

\begin{abstract}
Adventitial cystic disease is a rare peripheral arterial disease characterized by viscous and mucinous cysts in the arterial adventitia. Patients are usually young individuals without any risk factors for atherosclerosis. A 27-year-old man suffering from pain on his left leg was admitted. He had no history of atherosclerotic risk factors. Attenuated pulses were palpated. Doppler ultrasonography and computed tomography angiography revealed total occlusion in the femoral artery. Bypass surgery was performed using the vein graft. Histopathological examination revealed mucinous degeneration in the vascular wall. In conclusion, when arterial stenosis or occlusion develops in young patients having no risk factors for atherosclerosis, cystic adventitial disease should not be ignored. As frequently encountered in popliteal arteries, it must be distinguished carefully from popliteal artery entrapment syndrome.
\end{abstract}

Keywords: Cystic adventitial disease, peripheral arterial disease, vasculopathy.

Thromboangiitis obliterans, also known as Buerger disease, is most common obstructive peripheral arterial disease in young adults. Early atherosclerosis, popliteal entrapment syndrome, and vasculitis associated with claudication should be also considered in young adults. However, cystic adventitial disease is a rare peripheral arterial disorder and may lead to stenosis rather than occlusion. Cystic adventitial disease often affects the popliteal artery (about 85\%), typically in young or middle-aged men. It is characterized by a highly viscous and mucinous cyst located in the adventitia of an artery.

In this report, we present a patient with total occlusion in the common femoral artery due to cystic adventitial disease and discuss the cystic adventitial disease as an uncommon cause of peripheral arterial disease in the light of literature data.

\section{CASE REPORT}

A 27-year-old male patient presented to our department with an approximately two-year history of pain and coldness in his left leg. He had intermittent claudication after walking for 100 to 200 meters. He had no history of smoking, familial disease, or other atherosclerotic risk factors. Biochemical and hematological test results were within normal limits. On physical examination, his right lower extremity pulses were normal, while the left femoral, popliteal, and dorsalis pedis pulses were diminished and the left posterior tibial pulse was absent. All pulses in the left lower extremity gave monophasic continuous waves during examination with a pocket Doppler ultrasonography (USG). The ankle-brachial index at rest was 1.1 on the right and 0.6 on the left. A Duplex USG examination revealed a total

Received: August 15, 2019 Accepted: October 21, 2019 Published online: December 12, 2019

Correspondence: Erdinç Eroğlu, MD. Kahramanmaraş Sütçü İmam Üniversitesi Tıp Fakültesi Kalp ve Damar Cerrahisi Anabilim Dalı, 46040 0nikişubat, Kahramanmaraş, Türkiye. e-mail: erdinc046@hotmail.com 


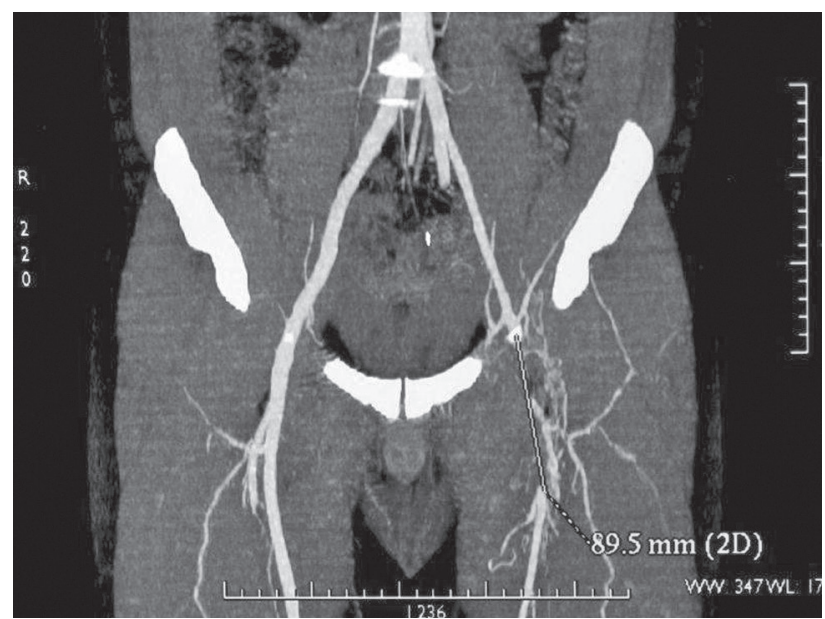

Figure 1. A computed tomography angiogram showing total occlusion in distally common femoral and proximal superficial arteries.

occlusion in the left common femoral and proximal superficial arteries and monophasic continuous waves in the popliteal artery and its continuity. A computed tomography $(\mathrm{CT})$ angiography showed a total occlusion in the common femoral and proximal superficial arteries in $10-\mathrm{cm}$ in size. Distal superficial and popliteal arteries were filled via collaterals (Figure 1). Based on these findings, an operation was planned. A written informed consent was obtained from the patient.

Operation was performed under spinal anesthesia. During surgery, the proximal common femoral artery and distal superficial femoral artery were explored. Normal arterial segment before the occlusion in the common femoral artery was reached and revascularization was performed with a reversed great saphenous vein graft interposition between the proximal common femoral artery and distal superficial femoral artery. During the operation, biopsy specimens were collected from the affected segment for histopathological examination which confirmed cystic adventitial disease as mucinous degeneration in the adventitial layer furnished with the endothelium (Figure 2).

The patient had an uneventful postoperative course and he remained asymptomatic at two years of follow-up.

\section{DISCUSSION}

Cystic adventitial disease is a rare vascular entity affecting the peripheral arteries. Although first described in the external iliac artery, the disease is more frequently located in the popliteal artery. In

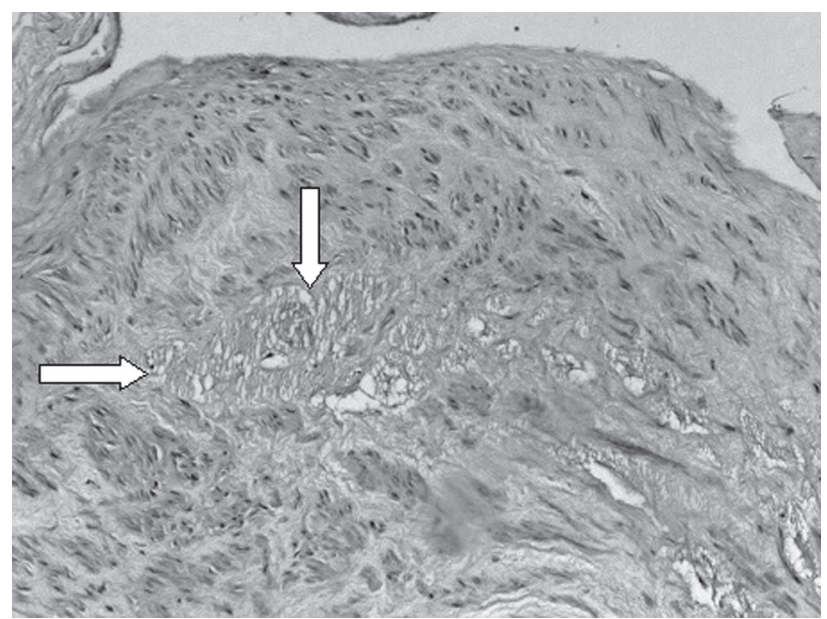

Figure 2. Mucinous degeneration in vascular wall furnished with endothelium (Alcian blue stain $\times 200$ ).

addition, femoral, radial, and ulnar arteries and some veins can be involved. ${ }^{[1-5]}$ In the clinical presentation, there is a cystic collection of the gelatinous fluid, containing mucoproteins and mucopolysaccharides, within the adventitial layer of a blood vessel. ${ }^{[1,3,6]}$ The cyst is located with tension between the adventitial and media layers of the vessel wall, and often leads to stenosis of the vascular lumen. ${ }^{[2,5]}$ As in our case, it may cause a total occlusion due to cyst enlargement.

Although the exact etiology of adventitial cystic disease is still unclear, some authors have proposed theories to elucidate its pathogenesis. The first one is the repetitive local trauma theory. Repeated stretch injuries cause degeneration of the arterial adventitia, suggesting that the arterial adventitial layer undergoes destruction with cystic degeneration due to repetitive trauma secondary to the movements occurring in the joint nearby. ${ }^{[3]}$ However, this does not explain why coronary artery disease (CAD) is also encountered in children and located far to the join and also fails to explain the occurrence of this condition in the veins. This theory is based on the proximity of the popliteal artery to the knee joint, resulting in the artery being subjected to repetitive flexion and extension. ${ }^{[3]}$ In our case, the disease involved the distal common femoral and proximal superficial femoral arteries which are relatively far to join and not undergo repetitive trauma.

Another theory, the developmental (embryological) theory, suggests that CAD is a manifestation of mucinsecreting cells derived from the mesenchyme of the adjacent join and incorrectly placed in the adventitia of the artery (and/or vein) during development. ${ }^{[3]}$ These cells secrete mucin later in life. The synovial theory suggests direct communication with the 
herniated synovial structures of the adjacent joint. ${ }^{[1,6]}$ In this situation, synovial cyst enlarges and tract along minor vascular branches to eventually implant in the adventitia of major vessel. ${ }^{[3]}$ The ganglion is the another theory in which the cyst is a true ganglion of the vessel wall. ${ }^{[3]}$ This theory is based on the similar findings in ganglia and a rich hyaluronic acid content of the adventitial cyst. However, other authors have not reported such biochemical findings and this theory must be considered speculative at best. ${ }^{[3]}$ The systemic disorders theory suggests that there is a degeneration and cyst formation of the adventitial layer as a part of a generalized connective tissue disease. ${ }^{[2,3,6]}$ Currently, the synovial and abnormal embryological development theories are the most widely adopted theories. ${ }^{[5]}$

In general, patients with cystic adventitial disease are young or middle-aged males with claudication in the absence of atherosclerotic disease. ${ }^{[4,5]}$ As this condition is uncommon, it is often unrecognized. Various non-invasive imaging modalities have been used for the diagnosis of CAD including color Doppler USG, CT, magnetic resonance imaging (MRI), CT angiography and magnetic resonance angiography (MRA). ${ }^{[1,2,4]}$ Color Doppler imaging shows arterial narrowing with increased peak systolic velocity in stenosis and demonstrates hypoechoic cystic lesions with or without multiple septated hypoechoic spaces. The CT and MRI can show cystic lesions and the relationship between vessels and CT angiography and MRA can demonstrate arterial stenosis or occlusion non-invasively and determine the inflow and the distal runoff of the arterial segment. ${ }^{[2]}$ In addition, Doppler USG, CT, and MRI are also performed for the differential diagnosis. We used Doppler USG and CT angiography in the presented case. A Duplex USG examination revealed a total occlusion in the left common femoral and proximal superficial arteries and CT angiography confirmed the sonographic findings.

If the artery is not entirely occluded, angiography shows a characteristic smooth tapering or curvilinear stenosis, referred to as the scimitar or hourglass sign, without post-stenotic dilatation and with no evidence of atherosclerotic disease. ${ }^{[1,2,7]}$ Arteriography has been regarded as the gold standard; however, it is diagnostic only when the study shows characteristic findings produced by the eccentric or concentric compression. However, in many cases when the angiographic findings are not typical or there is an arterial occlusion, arteriography is not conclusive and other imaging studies are required. ${ }^{[5]}$
In the treatment of CAD, surgery is preferred over endovascular or minimally invasive techniques due to significantly lower recurrence rates. Surgical treatment options to restore popliteal arterial flow include resection of the affected artery segment and interposition grafting with the autologous vein, aspiration of cystic contents, and resectional adventitial cystotomy. ${ }^{[2,4,6]}$ Surgical resection of the affected segment and bypass grafting seem to be the most appropriate treatment modalities. The prognosis is usually good after bypass grafting. ${ }^{[3,5]}$ Endovascular stent placement is often not recommended in these patients due to high recurrence rates.

In conclusion, when arterial stenosis or occlusion develops in young patients having no risk factors for atherosclerosis, cystic adventitial disease should not be ignored. As frequently encountered in popliteal arteries, it must be distinguished carefully from popliteal artery entrapment syndrome.

\section{Declaration of conflicting interests}

The authors declared no conflicts of interest with respect to the authorship and/or publication of this article.

\section{Funding}

The authors received no financial support for the research and/or authorship of this article.

\section{REFERENCES}

1. Hong JS, Lee KB, Kim DK, Kim DI. Cystic adventitial disease of the popliteal artery: report of a case. Surg Today 2007;37:719-22.

2. Papas TT, Georgiadis GS, Maltezos CK, Lazarides MK. Adventitial cystic disease of the popliteal artery: a potential cause of intermittent claudication. Wien Klin Wochenschr 2007;119:186-8.

3. Vasudevan A, Halak M, Lee S, Ong S, Nadkarni S. Cystic adventitial disease: a case report and literature review. ANZ J Surg 2005;75:1120-2.

4. Maged IM, Kron IL, Hagspiel KD. Recurrent cystic adventitial disease of the popliteal artery: successful treatment with percutaneous transluminal angioplasty. Vasc Endovascular Surg 2009;43:399-402.

5. Ortiz M WR, Lopera JE, Giménez CR, Restrepo S, Moncada R, Castañeda-Zúñiga WR. Bilateral adventitial cystic disease of the popliteal artery: a case report. Cardiovasc Intervent Radiol 2006;29:306-10.

6. Tomasian A, Lai C, Finn JP, Gelabert H, Krishnam MS. Cystic adventitial disease of the popliteal artery: features on 3T cardiovascular magnetic resonance. J Cardiovasc Magn Reson 2008;10:38.

7. Ishibashi S, Namiki K, Abe M, Shirahata Y, Ohnishi $\mathrm{K}$, Toyoda $\mathrm{T}$, et al. Cystic adventitial disease of the popliteal artery--a case of young boy. Tohoku J Exp Med 1995;176:173-80. 